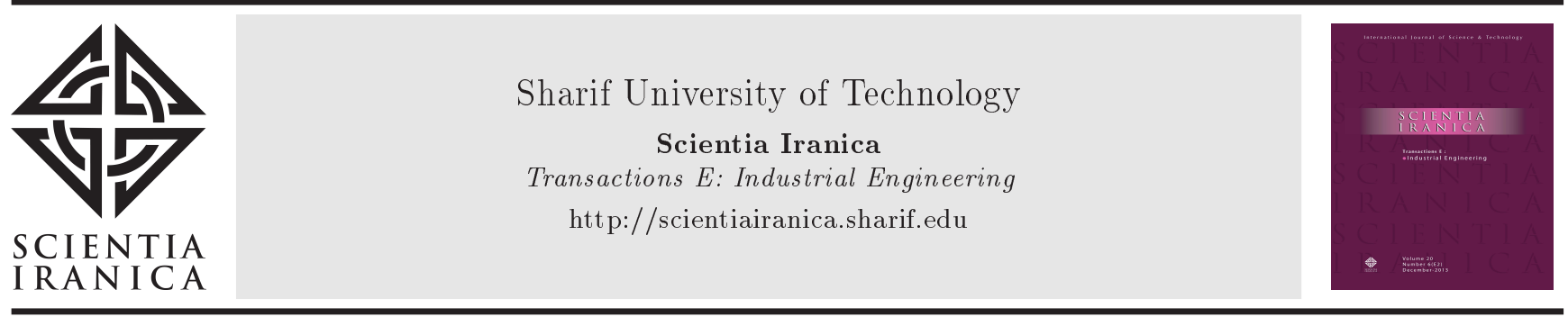

\title{
An efficient mixed-memory-type control chart for normal and non-normal processes
}

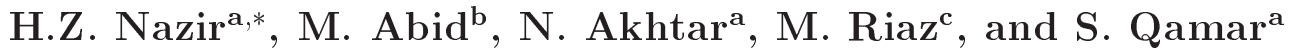 \\ a. Department of Statistics, University of Sargodha, Sargodha, 40100, Pakistan. \\ b. Department of Statistics, Government College University, Faisalabad, 38000, Pakistan. \\ c. Department of Mathematics and Statistics, King Fahad University of Petroleum and Minerals, Dhahran, 31261, Saudi Arabia.
}

Received 1 August 2018; received in revised form 21 April 2019; accepted 14 September 2019

\section{KEYWORDS}

Average run length; Control charts;

CUSUM;

Double EWMA;

Location parameter;

Memory-type charts.

\begin{abstract}
Statistical Process Control (SPC) techniques are commonly used to monitor process performance. Control charting technique is the most sophisticated tool of SPC and is categorized as memory-less and memory-type control charts. Shewhart-type control charts are of low efficiency in detecting small changes in the process parameters and are named as memory-less control charts. Memory-type control charts (e.g., Cumulative Sum (CUSUM) and Exponentially Weighted Moving Average (EWMA) charts) are very sensitive to small persistent shifts. In connection with enhancing the performance of CUSUM and EWMA charts, an efficient variant of memory-type charts for the location parameter is developed based on mixing the Double Exponentially Weighted Moving Average (DEWMA) chart and CUSUM chart by performing exponential smoothing twice. Performance of the proposed efficient variant is compared with existing counterparts under normal and nonnormal (heavy tails and skewed) environments. This study also provides an industrial application related to the monitoring of weights of quarters made by mint machine placed into service at U.S. Mint. From theoretical and numerical studies, it is revealed that the proposed variant of memory-type charts outperforms the counterparts in detecting shifts of small and moderate magnitudes.
\end{abstract}

2021 Sharif University of Technology. All rights reserved.

\section{Introduction}

Control charts, one of the key tools of Statistical Process Control (SPC), are categorized into two main types: memory-less and memory control charts. Shewhart charts, called memory-less control charts, are

\footnotetext{
*. Corresponding author. Tel.: 0092 3347711563

E-mail addresses: hafizzafarnazir@yahoo.com (H.Z. Nazir); mabid@gcuf.edu.pk (M. Abid);

noureen_akhtar31@hotmail.com (N. Akhtar);

riaz76qau@yahoo.com (M. Riaz); diyaqau@gmail.com (S. Qamar)
}

doi: $10.24200 /$ sci.2019.51437.2177 quite efficient in detecting larger shifts in the process location or dispersion. However, their efficiencies are under consideration in detecting small and moderate shifts in the process parameters. On the other hand, the memory control charts such as Cumulative Sum (CUSUM) control charts introduced by Page [1] and Exponentially Weighted Moving Average (EWMA) control charts suggested by Roberts [2] are more effective in detecting the small process shifts because they make use of the current as well as past sample information.

The most significant and commonly used measure to assess the performance of control charts is Average Run Length (ARL), which is simply the mean of the 
random variable Run Length $(\mathrm{RL})$. The $\mathrm{RL}$ is the number of samples and out-of-control signal is detected first. Some researchers have discouraged the only use of ARL due to the skewed behavior of its RL distribution (cf. [3-7]). Therefore, in order to explain the run length distribution further, it is better to report different important characteristics of the RL such as the Standard Deviation (SDRL) and some percentile points. When the process is in-control, the ARL is indicated by $\mathrm{ARL}_{0}$ and is expected to be large; if the process is out-of-control, the ARL is represented by $\mathrm{ARL}_{1}$ and is anticipated as small as possible.

To enhance the performance of CUSUM and EWMA charts, several amendments have been made (cf., [8-14]). Shamma and Shamma [15] proposed a control chart for evaluating the smaller and moderate shifts in the process mean using the method of Double Exponentially Weighted Moving Average (DEWMA) by performing exponential smoothing twice. Riaz et al. [16] improved the performance of CUSUM scheme in detecting small to large shifts employing the concept of runs rules scheme. Abbas et al. [17] proposed the implementation of different run rules for EWMA schemes. Abbas et al. [18] improved the design structure of EWMA and CUSUM control charts such that EWMA statistic would serve as the input for the CUSUM structure and be called as Mixed EWMACUSUM (MEC) chart. Zaman et al. [19] proposed a reverse version of the said MEC chart such that the CUSUM statistic would use the input for the EWMA structure and hereafter, named as Mixed CUSUMEWMA (MCE) chart. On the further development of the control charts using different approaches, the interested reader can see the work of Nazir et al. [20], Ahmad et al. [21], Riaz and Ali [22], Abujiya et al. [23], Abbasi et al. [24], Ajadi and Riaz [25], and Hussain et al. [26].

With the quality becoming more and more vital in today's industry and quality standards becoming higher and higher, a natural question that pops into mind is: "Is there a method to make the existing EWMA and CUSUM charts more sensitive to very small shifts in a process location parameter?" In this study, following Abbas et al. [18] and Zaman et al. [19], we explore such a possibility by combining features of the structures of CUSUM and DEWMA charts and propose an efficient chart by mixing the structures of CUSUM and DEWMA charts for the location parameter of the process. The control charts, designed under the assumption of normality, do not perform well under the violation of this assumption. Non-normal processes are more common in practice; hence, it is indispensable to develop the structure of the control charts under non-normality. Thus, the performance of the proposed control chart is under consideration in case of non-normal environments.
The rest of the paper is organized as follows: Section 2 presents the basic design structures of the CUSUM and DEWMA control charts and the proposed Efficient Variant (EV) scheme. Section 3 consists of design structure and derivation of the control limits of the proposed chart. Section 4 presents performance comparisons of the proposed scheme with its counterparts. Section 5 gives an industrial application of the proposed chart. At the end, Section 6 ends with conclusions.

\section{Description of CUSUM, DEWMA and the proposed charts}

Quality characteristic of interest, say $X$, is an independent sequence of observations $\left\{X_{t}\right\}(t=1,2,3, \cdots)$ following the normal distribution with mean $\mu_{0}+\delta \sigma_{0}$ and variance $\sigma_{0}^{2}$, i.e., $X_{t} \sim N\left(\mu_{0}+\delta \sigma_{0}, \sigma_{0}^{2}\right)$, where $\mu_{0}$ and $\sigma_{0}^{2}$ are the mean and variance of the process, respectively. The value of $\delta=0$ shows that the process is in-control; if not, the process mean shifts and the objective of the process monitoring is to detect the mean shift $\mu_{0}+\delta \sigma_{0}$ as early as possible following its occurrence. Without loss of generality, we assume that $\mu_{0}=0$ and $\sigma_{0}=1$. Thus, we assume the phaseII application of control charts with the in-control values of the parameters to be known. The following subsection contains details of the memory-type control charts.

\subsection{Cumulative Sum (CUSUM) chart}

Page [1] introduced the CUSUM chart employing the method of accumulating the positive and negative deviations from $\mu_{0}$ into two statistics $C_{i}^{+}$and $C_{i}^{-}$, respectively. These two statistics are defined as follows:

$$
\begin{aligned}
& C_{t}^{+}=\max \left[0,\left(X_{t}-\mu_{0}\right)-K+C_{t-1}^{+}\right], \\
& C_{t}^{-}=\max \left[0,-\left(X_{t}-\mu_{0}\right)-K+C_{t-1}^{-}\right],
\end{aligned}
$$

where $t$ is the sample number, $\mu_{0}$ is the target value, and $K$ is the reference or slack value which is commonly selected equal to half of the shift (in standard deviation unit) to be detected. The starting values of $C_{i}^{+}$and $C_{0}^{-}$are generally chosen equal to zero or the process location $\mu_{0}$, that is $C_{0}^{+}=C_{0}^{-}=\mu_{0}$, although it may be specified otherwise for a fast initial response (cf., [9]). The statistics $C_{i}^{+}$and $C_{i}^{-}$are plotted against the decision interval or control limit $H$ and the chart signals if either one of the statistics $\left(C_{0}^{+}\right.$or $\left.C_{i}^{-}\right)$exceeds the decision interval $H . K$ and $H$ are two parameters of the CUSUM chart that are defined as follows:

$$
K=k \times \sigma_{0}, \quad H=h \times \sigma_{0} .
$$

Here, $k$ and $h$ are the constants that are selected to fulfill a pre-defined $\mathrm{ARL}_{0}$ or according to the desired design conditions. 


\subsection{Double Exponentially Weighted Moving Average (DEWMA) chart}

Shamma and Shamma [15] proposed the DEWMA chart by performing exponential smoothing twice. The main disadvantage of EWMA statistic is that it always assigns strictly decreasing weights to historical data; however, this does not happen in case of DEWMA statistic (cf., [27]). The DEWMA statistic $Z_{t}$ is written as follows:

$$
\left.\begin{array}{l}
Y_{t}=\lambda_{1} X_{t}+\left(1-\lambda_{2}\right) Y_{t-1} \\
Z_{t}=\lambda_{3} Y_{t}+\left(1-\lambda_{4}\right) Z_{t-1}
\end{array}\right\},
$$

where $\lambda_{1}+\lambda_{2}=1, \lambda_{3}+\lambda_{4}=1$, and $\lambda_{1}$ and $\lambda_{3} \in$ $(0,1]$ are the smoothing parameters of the DEWMA chart. Also, as demonstrated by Zhang and Chen [27], the DEWMA statistic in Eq. (3) may be expressed as follows:

$$
\begin{aligned}
Z_{t}= & \lambda_{1} \lambda_{3} \sum_{j=1}^{t}\left\{\lambda_{2}^{t-j} \sum_{k=0}^{t-j}\left(\frac{\lambda_{4}}{\lambda_{2}}\right)^{k}\right\} X_{t} \\
& +\lambda_{3} \sum_{j=0}^{t-1} \lambda_{2}^{t-j} \lambda_{4}^{j} Y_{0}+\lambda_{4}^{t} Z_{0}, \quad t \geq 1 .
\end{aligned}
$$

If $\lambda_{1}=\lambda_{3}$, then:

$$
Z_{t}=\lambda_{1}^{2} \sum_{j=1}^{t}(t-j+1) \lambda_{2}^{t-j} X_{t}+t \lambda_{1} \lambda_{2}^{t} Y_{0}+\lambda_{2}^{t} Z_{0}
$$

Eq. (4) can be rewritten in the following if $\lambda_{1} \neq \lambda_{3}$ :

$$
\begin{aligned}
Z_{t}= & \lambda_{1} \lambda_{3} \sum_{j=1}^{t} \frac{1-\left(\lambda_{4} / \lambda_{2}\right)^{t-j+1}}{1-\left(\lambda_{4} / \lambda_{2}\right)} \lambda_{2}^{t-j} X_{t} \\
& +\lambda_{2} \lambda_{3} \frac{\lambda_{2}^{t}-\lambda_{4}^{t}}{\lambda_{2}-\lambda_{4}} Y_{0}+\lambda_{4}^{t} Z_{0} .
\end{aligned}
$$

The starting values of $Y_{t}$ and $Z_{t}$ are generally taken equal to the target values, i.e., $Y_{0}=Z_{0}=\mu_{0}$. The chart, DEWMA, signals if the statistic $Z_{t}$ falls beyond the following limits:

$$
\begin{aligned}
& L C L_{t}=\mu_{0}-L \sigma_{Z_{t}}, \\
& C L=\mu_{0}, \\
& U C L_{t}=\mu_{0}+L \sigma_{Z_{t}},
\end{aligned}
$$

where if $\lambda_{1}=\lambda_{3}$, then: $\sigma_{z t}$ is obtained by Eq. (8), shown in Box I, and if $\lambda_{1} \neq \lambda_{3}$, then: $\sigma_{z t}$ is obtained by Eq. (9), shown in Box II. The constant $L$ in Eq. (7) is the control limit coefficient and can carefully be chosen to satisfy the pre-specified $\mathrm{ARL}_{0}$ or, according to the design conditions, together with $\lambda_{1}$ and $\lambda_{3}$, control the performance of DEWMA chart.

\subsection{Proposed DEWMA-CUSUM chart}

To improve the design structure of EWMA and CUSUM control charts, Abbas et al. [18] and Zaman et al. [19] suggested the mixed versions of EWMA and CUSUM charts. The proposed chart is based on mixing the features of DEWMA and CUSUM charts by using the concept of double exponential smoothing, which makes the proposed chart sensitive to very small shifts in the process location parameter. The proposed mixed DEWMA-CUSUM chart, hereafter, is named as EV chart. The charting statistics $\left(E V_{t}^{+}\right.$and $\left.E V_{t}^{-}\right)$for this proposed $\mathrm{EV}$ chart are given as:

$$
\left.\begin{array}{c}
E V_{t}^{+}=\max \left[0,\left(Z_{t}-\mu_{0}\right)-P_{t}+E V_{t-1}^{+}\right] \\
E V_{t}^{-}=\max \left[0,-\left(Z_{t}-\mu_{0}\right)-P_{t}+E V_{t-1}^{-}\right]
\end{array}\right\},
$$

where $Z_{t}$ is defined as in Eq. (3) and $P_{t}$ is the reference value. The initial values for the statistics $E V_{0}^{+}$and $E V_{0}^{-}$are generally put equal to zero or the target value, $\mu_{0}$, i.e., $E V_{0}^{+}=E V_{0}^{-}=\mu_{0}$, although the initial values may be specified according to the desired design conditions. The statistics (given in Eq. (10)) are plotted alongside the control limit $Q_{t}$ and if either one of these statistics $\left(E V_{t}^{+}\right.$or $\left.E V_{t}^{-}\right)$goes outside the control limit $Q_{t}$, then the process is considered to be out-of-control; otherwise, it will be in-control. The standardized versions of $P_{t}$ and $Q_{t}$ are given as if $\lambda_{1}=\lambda_{3}$, then $P_{t}$ is obtained by Eq. (11), shown in

$$
\sigma_{Z_{t}}=\sqrt{\sigma_{0}^{2} \lambda_{1}^{4} \frac{1+\lambda_{2}^{2}-\left(t^{2}+2 t+1\right) \lambda_{2}^{2 t}+\left(2 t^{2}+2 t-1\right) \lambda_{2}^{2 t+2}-t^{2} \lambda_{2}^{2 t+4}}{\left(1-\lambda_{2}^{2}\right)^{3}}} .
$$

Box I

$$
\sigma_{Z_{t}}=\sqrt{\sigma_{0}^{2} \frac{\lambda_{1}^{2} \lambda_{3}^{2}}{\left(\lambda_{4}-\lambda_{2}\right)^{2}}\left\{\frac{\lambda_{4}^{2}\left(1-\lambda_{4}^{2 t}\right)}{1-\lambda_{4}^{2}}+\frac{\lambda_{2}^{2}\left(1-\lambda_{2}^{2 t}\right)}{1-\lambda_{2}^{2}}-2 \frac{\lambda_{2} \lambda_{4}\left(1-\left(\lambda_{2} \lambda_{4}\right)^{t}\right)}{1-\lambda_{2} \lambda_{4}}\right\}} .
$$




$$
P_{t}=p \times \sqrt{\sigma_{0}^{2} \lambda_{1}^{4} \frac{1+\lambda_{2}^{2}-\left(t^{2}+2 t+1\right) \lambda_{2}^{2 t}+\left(2 t^{2}+2 t-1\right) \lambda_{2}^{2 t+2}-t^{2} \lambda_{2}^{2 t+4}}{\left(1-\lambda_{2}^{2}\right)^{3}}} .
$$

\section{Box III}

$$
P_{t}=p \times \sqrt{\sigma_{0}^{2} \frac{\lambda_{1}^{2} \lambda_{3}^{2}}{\left(\lambda_{4}-\lambda_{2}\right)^{2}}\left\{\frac{\lambda_{4}^{2}\left(1-\lambda_{4}^{2 t}\right)}{1-\lambda_{4}^{2}}+\frac{\lambda_{2}^{2}\left(1-\lambda_{2}^{2 t}\right)}{1-\lambda_{2}^{2}}-2 \frac{\lambda_{2} \lambda_{4}\left(1-\left(\lambda_{2} \lambda_{4}\right)^{t}\right)}{1-\lambda_{2} \lambda_{4}}\right\}}
$$

Box IV

Box III, and if $\lambda_{1} \neq \lambda_{3}$, then $P_{t}$ is obtained by Eq. (12) shown in Box IV.

Unlike the usual CUSUM chart, the EV chart has time-varying reference values $P_{t}$ that result from the variance of DEWMA statistic in Eq. (8) and Eq. (9) and are functions of $\lambda_{1}$ and $\lambda_{3}$. The threshold control limit $Q_{t}$ is: if $\lambda_{1}=\lambda_{3}$, then $Q_{t}$ is obtained by Eq. (13), shown in Box V, and when $\lambda_{1} \neq \lambda_{3}$, then $Q_{t}$ is obtained by Eq. (14), shown in Box VI, where $p$ and $q$ are constants similar to $k$ and $h$ in Eq. (2), respectively, and can carefully be chosen to satisfy the pre-specified $\mathrm{ARL}_{0}$ or according to the design conditions. The flow chart of the proposed chart is provided in Figure 1.

\subsection{Derivation of the limits of the proposed scheme}

The construction of Phase-II control limits in Eqs. (13) and (14) of the proposed EV chart depends on the choice of the smoothing parameters, $\lambda_{1}$ and $\lambda_{3}$, the reference value, $P_{t}$, and the decision interval, $Q_{t}$. These parameters need to be chosen with care as these parameters control the performance of the proposed scheme. $q$ in Eqs. (13) and (14) is determined to obtain the desired $\mathrm{ARL}_{0}$ by setting $p=0.5$ as an optimal constant to detect a shift of size $\delta=1$, with different choices of $\lambda_{1}$ and $\lambda_{3}$, taking inspiration from Lucas [8], Shamma and Shamma [15], Abbas et al. [18], and Zaman et al. [19]. The values of $q$ to satisfy $\mathrm{ARL}_{0}=168,200,370$, and 500 are evaluated when $\lambda_{1}=\lambda_{3}$ and are given in Table 1 ; in addition, when $\lambda_{1} \neq \lambda_{3}$, values of $q$ to satisfy $\mathrm{ARL}_{0}=168$ are provided in Table 2 with their in-control SDRL values. The incontrol SDRL is also reported in Table 3 when $\lambda_{1}=\lambda_{3}$. Numerically, these values are hard to find and, hence, are determined using Monte Carlo simulation.

For a fixed in-control ARL $\left(\mathrm{ARL}_{0}\right)$, the values of $q$ increase as the smoothing parameters $\left(\lambda_{1}\right.$ and $\left.\lambda_{3}\right)$ tend to zero, and when $\lambda_{1}=\lambda_{3}$ approaches to one, this phenomenon occurs inversely (cf., Table 1 ). However, when a too small value of $\lambda_{1}=\lambda_{3}$ is used, the incontrol SDRL often becomes very large (cf., Table 3 ); on the contrary, when the values of $\lambda_{1}$ and $\lambda_{3}$ have the tendency to one, the in-control variability in the RL is small (cf., Table 2).

\section{Performance of the charts}

To evaluate the performance of the proposed EV chart,

$$
Q_{t}=q \times \sqrt{\sigma_{0}^{2} \lambda_{1}^{4} \frac{1+\lambda_{2}^{2}-\left(t^{2}+2 t+1\right) \lambda_{2}^{2 t}+\left(2 t^{2}+2 t-1\right) \lambda_{2}^{2 t+2}-t^{2} \lambda_{2}^{2 t+4}}{\left(1-\lambda_{2}^{2}\right)^{3}}} .
$$

Box V

$$
Q_{t}=q \times \sqrt{\sigma_{0}^{2} \frac{\lambda_{1}^{2} \lambda_{3}^{2}}{\left(\lambda_{4}-\lambda_{2}\right)^{2}}\left\{\frac{\lambda_{4}^{2}\left(1-\lambda_{4}^{2 t}\right)}{1-\lambda_{4}^{2}}+\frac{\lambda_{2}^{2}\left(1-\lambda_{2}^{2 t}\right)}{1-\lambda_{2}^{2}}-2 \frac{\lambda_{2} \lambda_{4}\left(1-\left(\lambda_{2} \lambda_{4}\right)^{t}\right)}{1-\lambda_{2} \lambda_{4}}\right\}}
$$




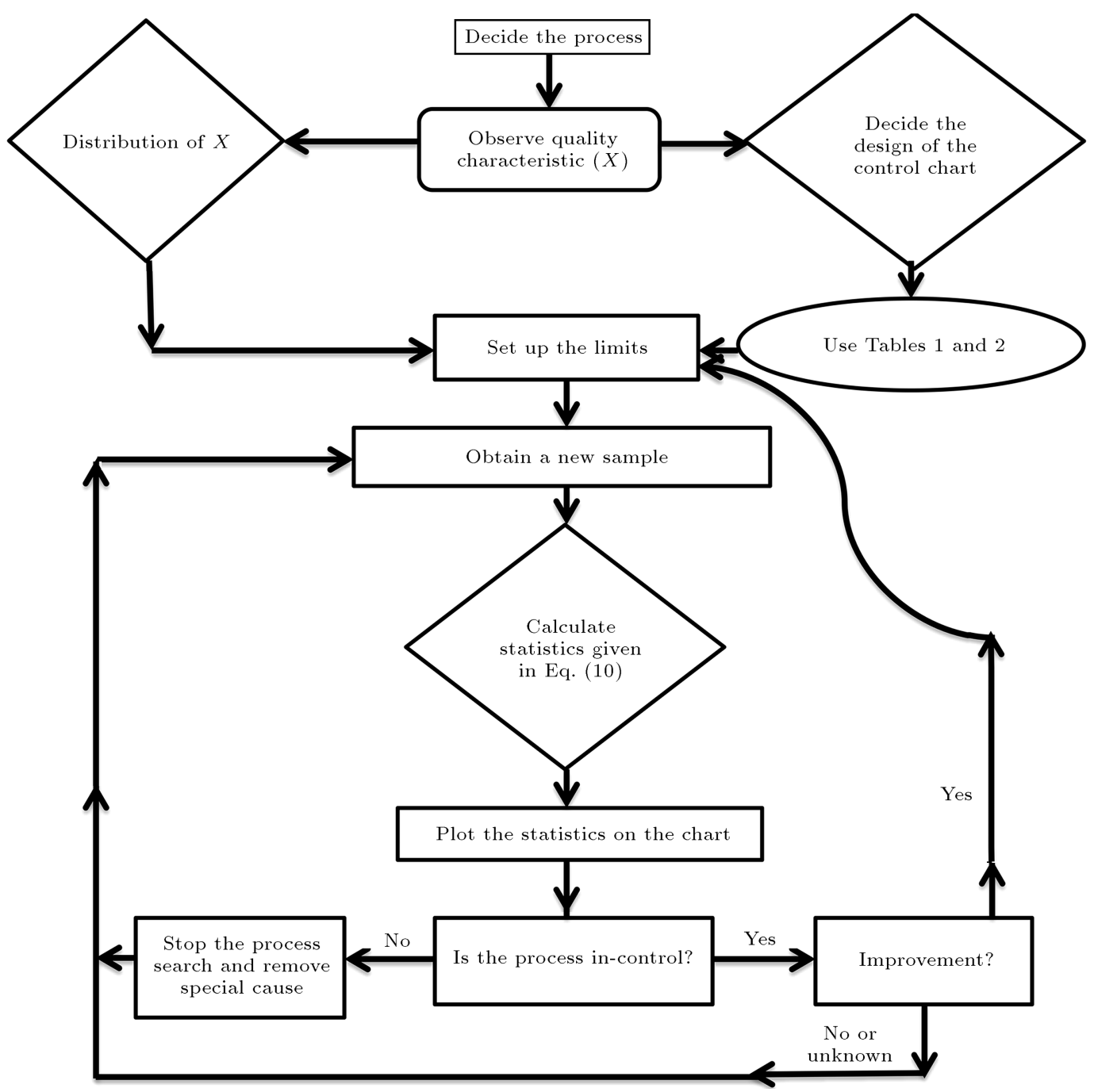

Figure 1. Flowchart of the proposed charts.

Table 1. $q$ Values of the Efficient Variant $(E V)$ chart for given $\mathrm{ARL}_{0}$ and $\lambda_{1}=\lambda_{3}$ with $p=0.5$.

\begin{tabular}{cccccccc}
\hline \multirow{2}{*}{$\mathbf{A R L}_{\mathbf{0}}$} & \multicolumn{7}{c}{$\boldsymbol{\lambda}_{\mathbf{1}}=\boldsymbol{\lambda}_{\mathbf{3}}$} \\
\cline { 2 - 8 } & $\mathbf{0 . 0 1}$ & $\mathbf{0 . 0 5}$ & $\mathbf{0 . 1}$ & $\mathbf{0 . 2 5}$ & $\mathbf{0 . 5}$ & $\mathbf{0 . 7 5}$ & $\mathbf{1}$ \\
\hline 168 & 116.04 & 51.34 & 39 & 23.91 & 13.1 & 7.4 & 4 \\
200 & 124 & 58.04 & 43.99 & 26 & 14 & 7.8 & 4.18 \\
370 & 165 & 84.6 & 60 & 32.8 & 17 & 9.24 & 4.78 \\
500 & 193.6 & 100.4 & 68.84 & 36.74 & 18.6 & 9.95 & 5.08 \\
\hline
\end{tabular}

Table 2. $q$ values of the Efficient Varient $(\mathrm{EV})$ chart for $\mathrm{ARL}_{0} \cong 168$ and $\lambda_{1} \neq \lambda_{3}$ with $p=0.5$.

\begin{tabular}{lccccccccc}
\hline$\lambda_{1}$ & 0.01 & 0.01 & 0.01 & 0.05 & 0.05 & 0.05 & 0.1 & 0.1 & 0.1 \\
$\lambda_{3}$ & 0.05 & 0.1 & 0.25 & 0.01 & 0.1 & 0.25 & 0.01 & 0.05 & 0.25 \\
$q$ & 38.28 & 30.06 & 25.89 & 150.84 & 38.28 & 30.3 & 169.4 & 55.9 & 28 \\
ARL & 169.79 & 168.33 & 167.09 & 168.46 & 168.90 & 169.70 & 169.31 & 169.07 & 169.04 \\
SDRL & 181.85 & 161.34 & 154.45 & 187.88 & 146.48 & 142.79 & 174.04 & 145.27 & 145.53 \\
$\lambda_{1}$ & 0.25 & 0.25 & 0.25 & 0.5 & 0.5 & 0.5 & 0.75 & 0.75 & 0.75 \\
$\lambda_{3}$ & 0.01 & 0.05 & 0.1 & 0.01 & 0.05 & 0.1 & 0.01 & 0.05 & 0.1 \\
$q$ & 193 & 61.26 & 39.8 & 209.7 & 66 & 40.68 & 218.6 & 68 & 41.5 \\
ARL & 168.61 & 168.35 & 169.66 & 169.31 & 167.88 & 169.10 & 168.55 & 168.17 & 168.88 \\
SDRL & 167.59 & 143.32 & 146.80 & 165.11 & 139.62 & 145.74 & 167.70 & 141.28 & 146.05 \\
\hline
\end{tabular}


Table 3. Standard Deviation of Run Length (SDRL) of the Efficient Varient (EV) chart for given $\mathrm{ARL}_{0}$ and $\lambda_{1}=\lambda_{3}$ with $p=0.5$.

\begin{tabular}{cccccccc}
\hline \multirow{2}{*}{ ARL $_{\mathbf{0}}$} & \multicolumn{7}{c}{$\boldsymbol{\lambda}_{\mathbf{1}}=\boldsymbol{\lambda}_{\mathbf{3}}$} \\
\cline { 2 - 8 } & $\mathbf{0 . 0 1}$ & $\mathbf{0 . 0 5}$ & $\mathbf{0 . 1}$ & $\mathbf{0 . 2 5}$ & $\mathbf{0 . 5}$ & $\mathbf{0 . 7 5}$ & $\mathbf{1}$ \\
\hline 168 & 252.46 & 149.18 & 143.49 & 148.73 & 154.85 & 159.21 & 161.73 \\
200 & 291.57 & 176.35 & 171.60 & 180.99 & 185.76 & 195.13 & 198.40 \\
370 & 417.52 & 317.56 & 324.32 & 336.72 & 351.91 & 359.34 & 371.45 \\
500 & 509.96 & 430.64 & 437.63 & 466.81 & 475.50 & 491.99 & 500.54 \\
\hline
\end{tabular}

Table 4. $\mathrm{ARL}_{1}$ and Standard Deviation of Run Length (SDRL) of Efficient Varient (EV) chart when $\lambda_{1}=\lambda_{3}$ with $\mathrm{ARL}_{0} \cong 168$.

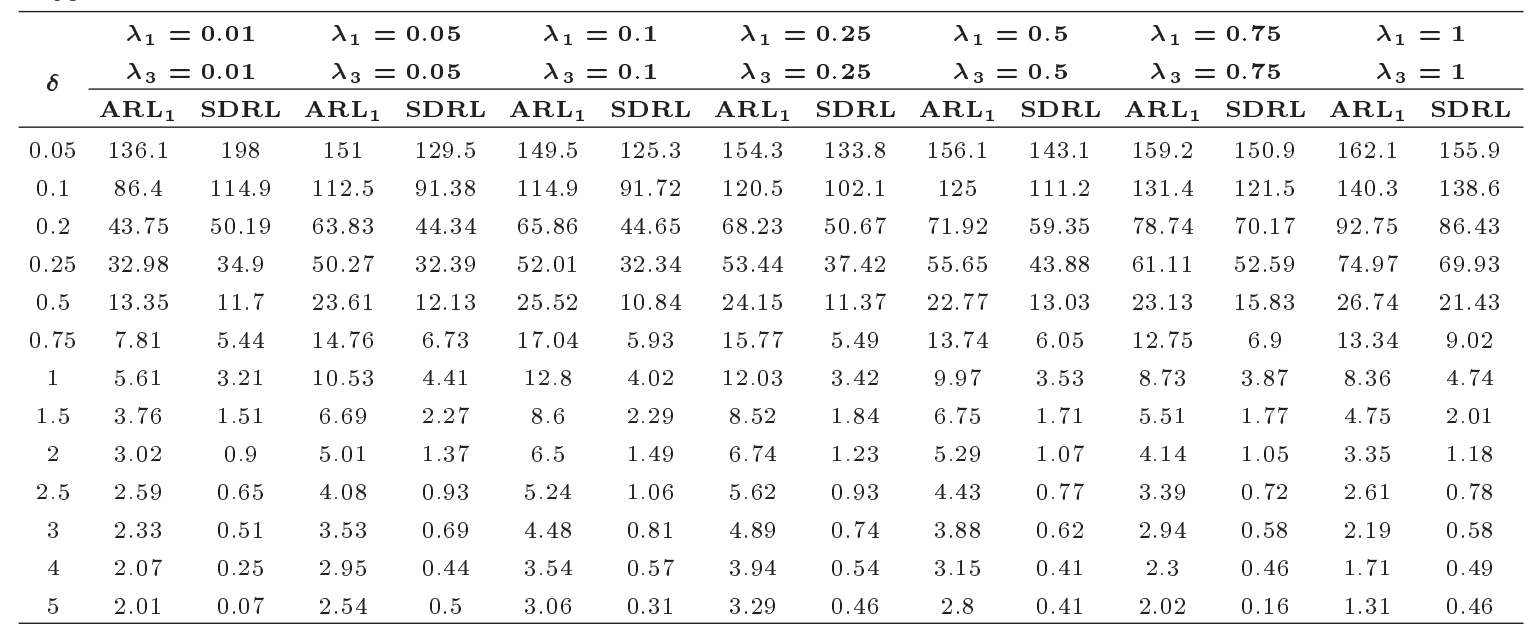

Table 5. ARL 1 and Standard Deviation of Run Length (SDRL) of Efficient Varient (EV) chart when $\lambda_{1}=\lambda_{3}$ with $\mathrm{ARL}_{0} \cong 200$.

\begin{tabular}{|c|c|c|c|c|c|c|c|c|c|c|c|c|c|c|}
\hline \multirow{2}{*}{$\delta$} & \multicolumn{2}{|c|}{$\begin{array}{l}\lambda_{1}=0.01 \\
\lambda_{3}=0.01\end{array}$} & \multicolumn{2}{|c|}{$\begin{array}{l}\lambda_{1}=0.05 \\
\lambda_{3}=0.05\end{array}$} & \multicolumn{2}{|c|}{$\begin{array}{l}\lambda_{1}=0.1 \\
\lambda_{3}=0.1\end{array}$} & \multicolumn{2}{|c|}{$\begin{array}{l}\lambda_{1}=0.25 \\
\lambda_{3}=0.25\end{array}$} & \multicolumn{2}{|c|}{$\begin{array}{l}\lambda_{1}=0.5 \\
\lambda_{3}=0.5\end{array}$} & \multicolumn{2}{|c|}{$\begin{array}{l}\lambda_{1}=0.75 \\
\lambda_{3}=0.75\end{array}$} & \multicolumn{2}{|c|}{$\begin{array}{l}\lambda_{1}=1 \\
\lambda_{3}=1\end{array}$} \\
\hline & $\mathbf{A R L}_{1}$ & SDRL & $\mathrm{ARL}_{1}$ & SDRL & $\mathrm{ARL}_{1}$ & SDRL & $\mathrm{ARL}_{1}$ & SDRL & $\mathrm{ARL}_{1}$ & SDRL & $\mathrm{ARL}_{1}$ & SDRL & $\mathrm{ARL}_{1}$ & SDRL \\
\hline 0.1 & 100.6 & 124.7 & 130.1 & 102.6 & 133.1 & 105.4 & 141.5 & 122.5 & 145.9 & 132.7 & 154.6 & 147.1 & 166.5 & 161.6 \\
\hline 0.2 & 47.98 & 50.92 & 70.8 & 46.38 & 72.91 & 48.37 & 74.72 & 55.6 & 79.3 & 65.58 & 87.41 & 78.2 & 108 & 103.9 \\
\hline 0.25 & 36.99 & 38.4 & 56.02 & 34.62 & 57.18 & 34.34 & 58.14 & 39.48 & 61.05 & 47.08 & 68.04 & 59.75 & 83.71 & 78.99 \\
\hline 0.75 & 8.4 & 5.88 & 16.55 & 7.07 & 18.75 & 6.1 & 16.62 & 5.63 & 14.62 & 6.44 & 13.23 & 6.94 & 13.97 & 9.41 \\
\hline 1 & 5.92 & 3.38 & 11.88 & 4.6 & 14.16 & 4.13 & 12.73 & 3.47 & 10.46 & 3.6 & 9.09 & 3.96 & 8.65 & 4.79 \\
\hline 1.5 & 3.95 & 1.55 & 7.49 & 2.4 & 9.55 & 2.38 & 9.02 & 1.88 & 7.04 & 1.74 & 5.7 & 1.82 & 4.95 & 2.04 \\
\hline 2 & 3.12 & 0.92 & 5.56 & 1.48 & 7.22 & 1.57 & 7.14 & 1.24 & 5.51 & 1.09 & 4.28 & 1.09 & 3.47 & 1.21 \\
\hline 2.5 & 2.69 & 0.68 & 4.49 & 1.02 & 5.81 & 1.13 & 5.97 & 0.93 & 4.63 & 0.78 & 3.51 & 0.76 & 2.72 & 0.81 \\
\hline
\end{tabular}

the ARL is used as a performance measure. Monte Carlo simulation is conducted to find $\mathrm{ARL}_{0}$ and $\mathrm{ARL}_{1}$ of the process. The simulation details are as follows: We have generated $10^{5}$ random observations from the distributions given in Section 2. The control limits of the EV chart are established using the expressions given in Eqs. (13) and (14) and the values of design parameters are given in Tables $1-3$. Then, we noted the number of sample points at which the plotting statistics $\left(E V_{t}^{+}\right.$or $\left.E V_{t}^{-}\right)$breach the control limits. At the end, we repeated this procedure $10^{5}$ times to get the distribution of the RL. The structure of the proposed scheme can easily be implemented in any statistical software. In this study, R language is utilized for the implementation to evaluate the properties of the charts. $\mathrm{ARL}_{1}$ and SDRL of EV chart for $\lambda_{1}=\lambda_{3}$ with fixed $\mathrm{ARL}_{0}=168,200,370,500$ are given in Tables $4-7$ and when $\lambda_{1} \neq \lambda_{3}, \mathrm{ARL}_{1}$ of the proposed chart with 
Table 6. $\mathrm{ARL}_{1}$ and Standard Deviation of Run Length (SDRL) of Efficient Varient (EV) chart when $\lambda_{1}=\lambda_{3}$ with $\mathrm{ARL}_{0} \cong 370$.

\begin{tabular}{|c|c|c|c|c|c|c|c|c|c|c|c|c|c|c|}
\hline \multirow[t]{2}{*}{$\delta$} & \multicolumn{2}{|c|}{$\begin{array}{l}\lambda_{1}=0.01 \\
\lambda_{3}=0.01\end{array}$} & \multicolumn{2}{|c|}{$\begin{array}{l}\lambda_{1}=0.05 \\
\lambda_{3}=0.05\end{array}$} & \multicolumn{2}{|c|}{$\begin{array}{l}\lambda_{1}=0.1 \\
\lambda_{3}=0.1\end{array}$} & \multicolumn{2}{|c|}{$\begin{array}{l}\lambda_{1}=0.25 \\
\lambda_{3}=0.25\end{array}$} & \multicolumn{2}{|c|}{$\begin{array}{l}\lambda_{1}=0.5 \\
\lambda_{3}=0.5\end{array}$} & \multicolumn{2}{|c|}{$\begin{array}{l}\lambda_{1}=0.75 \\
\lambda_{3}=0.75\end{array}$} & \multicolumn{2}{|c|}{$\begin{array}{l}\lambda_{1}=1 \\
\lambda_{3}=1\end{array}$} \\
\hline & $\mathbf{A R L}_{1}$ & SDRL & $\mathrm{ARL}_{1}$ & SDRL & $\mathbf{A R L}_{1}$ & SDRL & $\mathbf{A R L}_{1}$ & SDRL & $\mathrm{ARL}_{1}$ & SDRL & $\mathrm{ARL}_{1}$ & SDRL & $\mathrm{ARL}_{1}$ & SDRL \\
\hline 0.05 & 270.7 & 293.6 & 296.5 & 245.4 & 300.6 & 258 & 305.9 & 276.4 & 323.3 & 304.9 & 332.7 & 321.2 & 348.7 & 346.1 \\
\hline 0.1 & 157.1 & 152.4 & 192 & 144.2 & 196.4 & 156.6 & 208 & 177.5 & 228.9 & 208.6 & 252.6 & 240.9 & 283.5 & 276.3 \\
\hline 0.2 & 73.07 & 63.57 & 95.2 & 55.09 & 94.57 & 59.02 & 97.22 & 70.77 & 107.9 & 89.3 & 126.7 & 116.2 & 164.1 & 160.1 \\
\hline 0.25 & 53.93 & 45.04 & 74.81 & 38.36 & 73.35 & 40.56 & 73.08 & 40.10 & 78.7 & 61.37 & 92.52 & 81.4 & 123.2 & 117.5 \\
\hline 0.5 & 20.2 & 15.05 & 36.64 & 13.49 & 35.14 & 12.4 & 30.86 & 13.35 & 28.64 & 15.39 & 28.74 & 18.86 & 35.28 & 28.92 \\
\hline 0.75 & 11.29 & 7.13 & 23.94 & 7.77 & 23.86 & 6.53 & 19.88 & 6.31 & 16.91 & 6.89 & 15.65 & 7.98 & 16.34 & 10.66 \\
\hline 1 & 7.74 & 4.17 & 17.4 & 5.32 & 18.27 & 4.38 & 15.03 & 3.81 & 12.11 & 3.96 & 10.46 & 4.3 & 9.9 & 5.23 \\
\hline 1.5 & 4.89 & 1.92 & 11.03 & 2.97 & 12.53 & 2.58 & 10.62 & 2 & 8.1 & 1.91 & 6.49 & 1.94 & 5.52 & 2.18 \\
\hline 2 & 3.78 & 1.09 & 8.02 & 1.86 & 9.53 & 1.74 & 8.43 & 1.32 & 6.3 & 1.17 & 4.83 & 1.16 & 3.86 & 1.26 \\
\hline 2.5 & 3.18 & 0.74 & 6.28 & 1.29 & 7.65 & 1.29 & 7.04 & 0.99 & 5.24 & 0.83 & 3.94 & 0.8 & 3 & 0.85 \\
\hline 3 & 2.83 & 0.6 & 5.27 & 0.97 & 6.47 & 1 & 6.13 & 0.79 & 4.59 & 0.66 & 3.38 & 0.6 & 2.48 & 0.63 \\
\hline 4 & 2.35 & 0.48 & 4.07 & 0.62 & 4.97 & 0.68 & 4.91 & 0.59 & 3.73 & 0.52 & 2.73 & 0.48 & 1.96 & 0.4 \\
\hline 5 & 2.08 & 0.27 & 3.38 & 0.5 & 4.12 & 0.48 & 4.14 & 0.42 & 3.14 & 0.35 & 2.2 & 0.4 & 1.61 & 0.49 \\
\hline
\end{tabular}

Table 7. ARL $\mathrm{A}_{1}$ and Standard Deviation of Run Length (SDRL) of Efficient Varient (EV) chart when $\lambda_{1}=\lambda_{3}$ with $\mathrm{ARL}_{0} \cong 500$.

\begin{tabular}{|c|c|c|c|c|c|c|c|c|c|c|c|c|c|c|}
\hline \multirow[t]{2}{*}{$\delta$} & \multicolumn{2}{|c|}{$\begin{array}{l}\lambda_{1}=0.01 \\
\lambda_{3}=0.01\end{array}$} & \multicolumn{2}{|c|}{$\begin{array}{l}\lambda_{1}=0.05 \\
\lambda_{3}=0.05\end{array}$} & \multicolumn{2}{|c|}{$\begin{array}{l}\lambda_{1}=0.1 \\
\lambda_{3}=0.1\end{array}$} & \multicolumn{2}{|c|}{$\begin{array}{l}\lambda_{1}=0.25 \\
\lambda_{3}=0.25\end{array}$} & \multicolumn{2}{|c|}{$\begin{array}{l}\lambda_{1}=0.5 \\
\lambda_{3}=0.5\end{array}$} & \multicolumn{2}{|c|}{$\begin{array}{l}\lambda_{1}=0.75 \\
\lambda_{3}=0.75\end{array}$} & \multicolumn{2}{|c|}{$\begin{array}{l}\lambda_{1}=1 \\
\lambda_{3}=1\end{array}$} \\
\hline & $\mathbf{A R L}_{1}$ & SDRL & $\mathrm{ARL}_{1}$ & SDRL & $\mathrm{ARL}_{1}$ & SDRL & $\mathrm{ARL}_{1}$ & SDRL & $\mathrm{ARL}_{1}$ & SDRL & $\mathrm{ARL}_{1}$ & SDRL & $\mathrm{ARL}_{1}$ & SDRL \\
\hline 0.05 & 349.7 & 335.3 & 378.4 & 312 & 384.2 & 330.4 & 405.2 & 369.5 & 430.3 & 410.7 & 444.3 & 430 & 461.3 & 455.8 \\
\hline 0.1 & 195.1 & 165.9 & 228.6 & 169.3 & 233.9 & 185.4 & 258.3 & 223.5 & 288.1 & 268.6 & 315.1 & 307.2 & 374 & 376.3 \\
\hline 0.2 & 89.08 & 67.7 & 109.3 & 61.59 & 106.8 & 65.55 & 112 & 82.12 & 125 & 104.3 & 150 & 136.2 & 199.2 & 188.4 \\
\hline 0.25 & 66.05 & 48.95 & 85.54 & 41.27 & 82.09 & 43.82 & 81.69 & 52.68 & 89.02 & 69.04 & 105.2 & 91.68 & 145.8 & 137.3 \\
\hline 0.5 & 24.64 & 16.64 & 41.97 & 13.89 & 38.87 & 13 & 33.57 & 14.23 & 31.09 & 16.37 & 31.37 & 20.26 & 38.91 & 31.43 \\
\hline 0.75 & 13.59 & 8.03 & 28.16 & 8.12 & 26.47 & 6.75 & 21.72 & 6.7 & 18.22 & 7.23 & 16.6 & 8.28 & 17.35 & 11.26 \\
\hline 1 & 9.16 & 4.74 & 20.61 & 5.53 & 20.38 & 4.51 & 16.31 & 3.97 & 13 & 4.15 & 11.13 & 4.47 & 10.53 & 5.57 \\
\hline 1.5 & 5.64 & 2.16 & 13.14 & 3.18 & 14.1 & 2.65 & 11.46 & 2.07 & 8.64 & 1.99 & 6.9 & 2.03 & 5.86 & 2.27 \\
\hline 2 & 4.24 & 1.24 & 9.52 & 2.06 & 10.77 & 1.81 & 9.1 & 1.36 & 6.7 & 1.21 & 5.13 & 1.21 & 4.08 & 1.3 \\
\hline 2.5 & 3.51 & 0.8 & 7.47 & 1.45 & 8.66 & 1.35 & 7.65 & 1.02 & 5.56 & 0.86 & 4.14 & 0.83 & 3.14 & 0.88 \\
\hline 3 & 3.11 & 0.6 & 6.16 & 1.08 & 7.31 & 1.05 & 6.63 & 0.82 & 4.86 & 0.68 & 3.56 & 0.64 & 2.61 & 0.66 \\
\hline 4 & 2.6 & 0.51 & 4.68 & 0.69 & 5.58 & 0.72 & 5.31 & 0.59 & 3.96 & 0.47 & 2.88 & 0.44 & 2.03 & 0.39 \\
\hline 5 & 2.22 & 0.41 & 3.89 & 0.5 & 4.58 & 0.56 & 4.47 & 0.52 & 3.32 & 0.47 & 2.35 & 0.48 & 1.72 & 0.45 \\
\hline
\end{tabular}

fixed $\mathrm{ARL}_{0}=168$ is provided in Table 8. The following observations can be made in Tables $4-8$ :

i. The detection ability of the proposed chart for small shifts is greater for small values of smoothing parameters than the large choices of $\lambda_{1}$ and $\lambda_{3}$. To detect the shift of size $\delta=0.25$, the average run length is much lower for $\lambda_{1}=\lambda_{3}=0.01$ than any other choices of the smoothing parameter (cf., Table 4);

ii. The performance of the EV chart is substantial at smaller values of $\lambda_{1}=\lambda_{3}$;

iii. With the moderate value of $\lambda_{1}=\lambda_{3}=0.10$, the shift of size $\delta=0.50$ can be identified with smaller variability in the run length distribution;

iv. When $\lambda_{1} \neq \lambda_{3}$, the efficient choices of $\lambda_{1}$ and $\lambda_{3}$ for quickly detecting $\delta=0.50$ are to use $\lambda_{1}=0.05$ and $\lambda_{3}=0.01$ along with the choices of $\lambda_{1}=0.1$ and $\lambda_{3}=0.01$; v. The proposed chart works efficiently in finding an undesirable process level with $\lambda_{1}=\lambda_{3}$, as compared to the chart with $\lambda_{1} \neq \lambda_{3}$;

vi. The recommendation is to use $0<\lambda_{1}=\lambda_{3}<0.25$ for quick detection of shifts of magnitude, i.e., $\delta=$ 0.50 (cf., Tables $4-7$ ), and in case of $\lambda_{1} \neq \lambda_{3}$ to better select $0.05 \leq \lambda_{1} \leq 0.1$ and $\lambda_{3}=0.01$.

\section{Comparisons with other mixed charts under normal environment}

The objective here is to provide an efficient chart from the existing mixed charts, e.g., MEC and MCE charts. To this end, we compare the performance of the EV chart, only, with those of MEC and MCE charts because the papers that have already investigated MEC and MCE charts provide detailed comparisons with some other charts. For valid comparisons, the EV, $\mathrm{MEC}$, and MCE charts have been given the same in- 
Table 8. $\mathrm{ARL}_{1}$ of Efficient Varient (EV) chart when $\lambda_{1} \neq \lambda_{3}$ with $\mathrm{ARL}_{0} \cong 168$.

\begin{tabular}{|c|c|c|c|c|c|c|c|c|c|}
\hline \multirow{2}{*}{$\delta$} & \multicolumn{3}{|c|}{$\lambda_{1}=0.01$} & \multicolumn{3}{|c|}{$\lambda_{1}=0.05$} & \multicolumn{3}{|c|}{$\lambda_{1}=0.10$} \\
\hline & $\lambda_{3}=0.05$ & $\lambda_{3}=0.10$ & $\lambda_{3}=0.25$ & $\lambda_{3}=0.01$ & $\lambda_{3}=0.10$ & $\lambda_{3}=0.25$ & $\lambda_{3}=0.01$ & $\lambda_{3}=0.05$ & $\lambda_{3}=0.25$ \\
\hline 0.05 & 143.7 & 147.1 & 147.9 & 144.9 & 151.2 & 151.3 & 146.5 & 148.7 & 150.4 \\
\hline 0.1 & 103.6 & 108.9 & 110.5 & 100.8 & 113.9 & 114.5 & 105.9 & 114.6 & 117.6 \\
\hline 0.2 & 57.24 & 61.29 & 63.53 & 53.71 & 65.64 & 66.36 & 56.62 & 65.1 & 66.47 \\
\hline 0.25 & 44.15 & 48.97 & 51.39 & 40.65 & 52.19 & 52.82 & 43.69 & 51.78 & 52.9 \\
\hline 0.5 & 19.37 & 23.49 & 26.6 & 16.7 & 26.26 & 27.55 & 17.9 & 24.07 & 25.76 \\
\hline 0.75 & 11.84 & 15.34 & 18.69 & 9.75 & 17.59 & 19.46 & 10.67 & 15.39 & 18 \\
\hline 1 & 8.2 & 11.11 & 14.45 & 6.86 & 13.16 & 15.35 & 7.39 & 10.96 & 14.02 \\
\hline 1.5 & 5.27 & 7.25 & 10.24 & 4.47 & 8.75 & 11.09 & 4.78 & 7.01 & 10.1 \\
\hline 2 & 4.04 & 5.44 & 8.03 & 3.5 & 6.58 & 8.79 & 3.72 & 5.24 & 8.02 \\
\hline 2.5 & 3.38 & 4.43 & 6.65 & 2.98 & 5.29 & 7.29 & 3.16 & 4.28 & 6.71 \\
\hline 3 & 2.98 & 3.79 & 5.7 & 2.65 & 4.51 & 6.31 & 2.81 & 3.67 & 5.79 \\
\hline 4 & 2.45 & 3.09 & 4.5 & 2.23 & 3.55 & 5 & 2.33 & 3.04 & 4.61 \\
\hline 5 & 2.11 & 2.7 & 3.79 & 2.04 & 3.06 & 4.18 & 2.07 & 2.68 & 3.9 \\
\hline \multirow{2}{*}{$\delta$} & \multicolumn{3}{|c|}{$\lambda_{1}=0.25$} & \multicolumn{3}{|c|}{$\lambda_{1}=0.50$} & \multicolumn{3}{|c|}{$\lambda_{1}=0.75$} \\
\hline & $\lambda_{3}=0.01$ & $\lambda_{3}=0.05$ & $\lambda_{3}=0.10$ & $\lambda_{3}=0.01$ & $\lambda_{3}=0.05$ & $\lambda_{3}=0.25$ & $\lambda_{3}=0.01$ & $\lambda_{3}=0.05$ & $\lambda_{3}=0.25$ \\
\hline 0.05 & 145.6 & 146.9 & 148.5 & 149.8 & 153.9 & 153.5 & 148.2 & 152.2 & 155 \\
\hline 0.1 & 107 & 114.3 & 117 & 108.1 & 118 & 118.7 & 106.8 & 117.4 & 120 \\
\hline 0.2 & 58.05 & 66.27 & 67.3 & 58.34 & 67.63 & 67.98 & 57.97 & 67.54 & 68.87 \\
\hline 0.25 & 45.1 & 52.75 & 53.34 & 44.87 & 53.58 & 53.72 & 44.62 & 53.56 & 54.29 \\
\hline 0.5 & 18.55 & 24.3 & 24.8 & 18.67 & 24.96 & 24.91 & 18.52 & 25.05 & 25.15 \\
\hline 0.75 & 10.9 & 15.27 & 16.16 & 10.89 & 15.55 & 15.93 & 10.77 & 15.6 & 16.03 \\
\hline 1 & 7.68 & 10.98 & 12.02 & 7.64 & 11.08 & 11.69 & 7.51 & 11.07 & 11.67 \\
\hline 1.5 & 5.03 & 7.12 & 8.12 & 4.93 & 7.05 & 7.71 & 4.8 & 6.96 & 7.61 \\
\hline 2 & 3.87 & 5.33 & 6.16 & 3.82 & 5.25 & 5.83 & 3.7 & 5.12 & 5.68 \\
\hline 2.5 & 3.3 & 4.36 & 5.04 & 3.22 & 4.25 & 4.73 & 3.1 & 4.11 & 4.56 \\
\hline 3 & 2.92 & 3.75 & 4.31 & 2.87 & 3.67 & 4.08 & 2.75 & 3.53 & 3.91 \\
\hline 4 & 2.44 & 3.09 & 3.44 & 2.39 & 3.04 & 3.27 & 2.3 & 2.93 & 3.15 \\
\hline 5 & 2.11 & 2.75 & 3.02 & 2.09 & 2.69 & 2.92 & 2.06 & 2.52 & 2.8 \\
\hline
\end{tabular}

Table 9. ARLs of Mixed EWMA-CUSUM (MEC), and Mixed CUSUM-EWMA (MCE) charts with $\mathrm{ARL}_{0} \cong 168$.

\begin{tabular}{|c|c|c|c|c|c|c|c|c|}
\hline \multirow{2}{*}{$\delta$} & \multicolumn{4}{|c|}{ MEC chart } & \multicolumn{4}{|c|}{ MCE chart } \\
\hline & $\lambda=0.1$ & $\lambda=0.25$ & $\lambda=0.5$ & $\lambda=0.75$ & $\lambda=0.1$ & $\lambda=0.25$ & $\lambda=0.5$ & $\lambda=0.75$ \\
\hline 0 & 168.04 & 168.07 & 169.88 & 171.04 & 168.30 & 169.39 & 168.36 & 170.28 \\
\hline 0.25 & 52.64 & 54.18 & 59.78 & 68.15 & 67.79 & 70.42 & 72.90 & 73.45 \\
\hline 0.5 & 24.86 & 22.41 & 22.55 & 24.13 & 25.61 & 24.94 & 25.61 & 25.43 \\
\hline 0.75 & 17.02 & 14.02 & 12.86 & 12.61 & 13.52 & 12.77 & 12.64 & 12.43 \\
\hline 1 & 13.33 & 10.48 & 8.96 & 8.27 & 9.34 & 8.39 & 7.78 & 7.54 \\
\hline 1.5 & 9.74 & 7.33 & 5.79 & 5.00 & 5.87 & 5.05 & 4.42 & 4.09 \\
\hline 2 & 7.91 & 5.82 & 4.43 & 3.74 & 4.41 & 3.73 & 3.14 & 2.79 \\
\hline
\end{tabular}

control average run length $\left(\mathrm{ARL}_{0}\right)$ and then, their respective out-of-control average run lengths $\left(\mathrm{ARL}_{1}\right)$ are compared. For the aforesaid purpose, the ARLs of the MEC and MCE charts are simulated. Some representative results are provided in Table 9 .

According to Tables 4 and 9, EV chart is slightly proficient than the MEC chart, but outperforms MCE chart in detecting small to moderate changes in the process location parameter when the smoothing parameters of $\mathrm{EV}$ chart are equal, i.e., $\lambda_{1}=\lambda_{3}$. The performance of the proposed chart is more obvious and substantial at larger values of $\lambda_{1}=\lambda_{3}$.
According to the comparison of the results of $\mathrm{EV}$ chart shown in Table 4 having $\lambda_{1} \neq \lambda_{3}$ with those of the MEC and MCE charts shown in Table 9, it can be observed that the EV chart is even more sensitive to small shifts. The above discussion is made when $\mathrm{ARL}_{0} \cong 168$, but this is generally true when other incontrol ARLs are considered.

According to Section 2, the only use of ARL has been criticized by many researchers due to its skewed behavior. Therefore, to facilitate a better understanding of the RL distribution of $\mathrm{EV}, \mathrm{MEC}$, and MCE charts, some other measures such as SDRL and differ- 
Table 10. Characteristics of in-control run length for Efficient Variant (EV), Mixed EWMA-CUSUM (MEC), and Mixed CUSUM-EWMA (MCE) charts with $\mathrm{ARL}_{0} \cong 168$.

\begin{tabular}{ccccc}
\hline Characteristics & $\begin{array}{c}\mathbf{E V} \\
\left(\boldsymbol{\lambda}_{\mathbf{1}}=\boldsymbol{\lambda}_{\mathbf{3}}=\mathbf{0 . 1}\right)\end{array}$ & $\begin{array}{c}\mathbf{E V} \\
\left(\boldsymbol{\lambda}_{\mathbf{1}}=\mathbf{0 . 1}, \boldsymbol{\lambda}_{\mathbf{3}}=\mathbf{0 . 0 1}\right)\end{array}$ & $\begin{array}{c}\text { MEC } \\
(\boldsymbol{\lambda}=\mathbf{0 . 1})\end{array}$ & $\begin{array}{c}\text { MCE } \\
(\boldsymbol{\lambda}=\mathbf{0 . 1})\end{array}$ \\
\hline Min & 5 & 2 & 9 & 3 \\
$P_{1}$ & 17 & 4 & 18 & 8 \\
$P_{5}$ & 38 & 7 & 36 & 25 \\
$P_{10}$ & 47 & 13 & 45 & 35 \\
$P_{25}$ & 66 & 41 & 64 & 55 \\
$P_{50}$ & 125 & 116 & 125 & 122 \\
$P_{75}$ & 222 & 239 & 229 & 234 \\
$P_{90}$ & 356 & 399 & 368 & 382 \\
$P_{95}$ & 457 & 521 & 470 & 494 \\
$P_{99}$ & 688 & 792 & 699 & 741 \\
Max & 1746 & 1879 & 2282 & 1796 \\
ARL & 167.67 & 169.24 & 170.60 & 170.40 \\
SDRL & 143.72 & 174.73 & 149.58 & 161.94 \\
\hline
\end{tabular}

ent percentiles $\left(P_{i}, i\right.$ th percentile) along with the smallest and largest RL of the in-control process are reported in Table 10 and these measures help study the short run and long run behaviors of the RL distribution. For instance, the $5 \%$ percentiles of the RL distribution of the EV, MEC, and MCE charts are on average about $17,4,18$, and 8 observations (cf., Table 10).

To get a deeper insight into the out-of-control RL distribution, Figure 2 presents the RL distribution curves of all the charts considering the value of smoothing parameter equal to 0.10 with $\delta=0.25$ under a normal environment. The curves give the cumulative probability of detecting an out-of-control situation. A higher curve shows the superiority of a chart in terms of its quick detection of shifts in the process parameter.

According to Figure 2, EV chart has higher probabilities for small RL to detect the shift than that

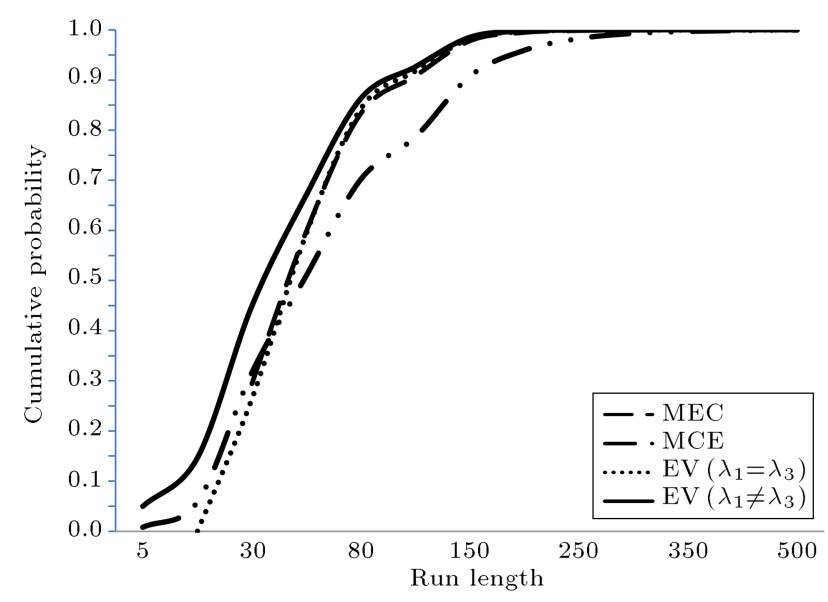

Figure 2. Run length curves of Efficient Variant (EV), Mixed EWMA-CUSUM (MEC), and Mixed CUSUM-EWMA (MCE) charts with $\delta=0.25$ and $\mathrm{ARL}_{0} \cong 168$. of other memory charts. To detect a shift of magnitude $\delta=0.25$ at a RL equal to 30 , the practitioner needs to spend a shorter span of time with the mixed EV chart than MEC and MCE charts.

Overall, the smaller values of smoothing parameters of the proposed EV chart pointed to its better performance in spotting smaller changes in the process location parameter.

\subsection{Evaluation under non-normal environments}

Design and implementation of the proposed EV chart discussed in the preceding subsection are based on the assumptions that process measurements are independent and identically distributed, both the in-control and out-of-control distributions are normal, and the process parameters of the in-control distribution are known. However, there are many practical situations where these assumptions can be invalid. The next section discusses effects on the performance of the proposed EV chart of the case when the process measurements collected in different time periods are from non-normal environments. For the sake of comparisons, the counterpart charts of MEC and MCE are also considered.

\subsubsection{Limits based on normality}

In this sub-section, the impact of non-normal observations on EV, MEC, and MCE charts with control limits based on normality is evaluated. Consider the following scenario: When process measurements follow a nonnormal distribution, i.e., $t$ distribution with 4 degrees of freedom $\left(t_{4}\right)$ having heavy tails and being flatter than that of normal distribution. According to Table 11, it can be observed that the proposed EV and MEC charts are insensitive to changes in the environment (i.e., $t_{4}$ ) 
Table 11. Characteristics of run length distribution of mixed charts under uncorrected limits with $\mathrm{ARL}_{0} \cong 168$.

\begin{tabular}{|c|c|c|c|c|c|c|c|c|c|c|c|c|c|}
\hline Chart & $\begin{array}{l}\text { Smoothing } \\
\text { parameter }\end{array}$ & $\begin{array}{c}\text { Reference } \\
\text { value }\end{array}$ & Limit & $\delta$ & ARL & SDRL & Min & $P_{5}$ & $P_{25}$ & $P_{50}$ & $P_{75}$ & $P_{95}$ & Max \\
\hline \multirow{14}{*}{$\mathrm{EV}$} & \multirow{14}{*}{0.1} & \multirow{14}{*}{0.5} & \multirow{14}{*}{39} & 0 & 173.4 & 151.33 & 2 & 28 & 68 & 129 & 230 & 470 & 1542 \\
\hline & & & & 0.05 & 153.55 & 130.5 & 2 & 27 & 62 & 115 & 205 & 410 & 1655 \\
\hline & & & & 0.1 & 117.94 & 94.52 & 2 & 25 & 51 & 90 & 155 & 302 & 959 \\
\hline & & & & 0.2 & 66.45 & 44.93 & 3 & 19 & 35 & 54 & 85 & 156 & 455 \\
\hline & & & & 0.25 & 52.65 & 32.39 & 2 & 18 & 30 & 44 & 67 & 116 & 317 \\
\hline & & & & 0.5 & 25.33 & 10.5 & 2 & 12 & 18 & 24 & 31 & 45 & 91 \\
\hline & & & & 0.75 & 16.95 & 5.81 & 2 & 9 & 13 & 16 & 20 & 28 & 63 \\
\hline & & & & 1 & 12.78 & 3.92 & 2 & 7 & 10 & 12 & 15 & 20 & 38 \\
\hline & & & & 1.5 & 8.54 & 2.22 & 2 & 5 & 7 & 8 & 10 & 12 & 26 \\
\hline & & & & 2 & 6.45 & 1.43 & 2 & 4 & 6 & 6 & 7 & 9 & 15 \\
\hline & & & & 2.5 & 5.26 & 1.04 & 2 & 4 & 5 & 5 & 6 & 7 & 12 \\
\hline & & & & 3 & 4.49 & 0.8 & 2 & 3 & 4 & 4 & 5 & 6 & 11 \\
\hline & & & & 4 & 3.54 & 0.59 & 2 & 3 & 3 & 4 & 4 & 4 & 9 \\
\hline & & & & 5 & 3.05 & 0.32 & 2 & 3 & 3 & 3 & 3 & 4 & 7 \\
\hline \multirow{14}{*}{$\mathrm{MCE}$} & \multirow{14}{*}{0.1} & \multirow{14}{*}{0.5} & \multirow{14}{*}{5.96} & 0 & 154.49 & 149.85 & 1 & 12 & 47 & 109 & 214 & 448 & 1397 \\
\hline & & & & 0.05 & 145.05 & 137.97 & 1 & 13 & 46 & 103 & 199 & 422 & 1329 \\
\hline & & & & 0.1 & 131.27 & 127.7 & 1 & 12 & 42 & 92 & 178 & 382 & 1391 \\
\hline & & & & 0.2 & 93.43 & 85.78 & 1 & 10 & 32 & 67 & 129 & 271 & 829 \\
\hline & & & & 0.25 & 74.17 & 67.38 & 1 & 9 & 26 & 54 & 100 & 211 & 751 \\
\hline & & & & 0.5 & 27.18 & 20.48 & 1 & 7 & 13 & 21 & 35 & 67 & 226 \\
\hline & & & & 0.75 & 13.7 & 7.69 & 1 & 5 & 8 & 12 & 17 & 29 & 78 \\
\hline & & & & 1 & 9.09 & 4.04 & 1 & 4 & 6 & 8 & 11 & 17 & 37 \\
\hline & & & & 1.5 & 5.48 & 1.88 & 1 & 3 & 4 & 5 & 6 & 9 & 18 \\
\hline & & & & 2 & 3.99 & 1.17 & 1 & 2 & 3 & 4 & 5 & 6 & 11 \\
\hline & & & & 2.5 & 3.16 & 0.86 & 1 & 2 & 3 & 3 & 4 & 5 & 8 \\
\hline & & & & 3 & 2.6 & 0.7 & 1 & 2 & 2 & 3 & 3 & 4 & 6 \\
\hline & & & & 4 & 1.95 & 0.46 & 1 & 1 & 2 & 2 & 2 & 3 & 5 \\
\hline & & & & 5 & 1.47 & 0.52 & 1 & 1 & 1 & 1 & 2 & 2 & 4 \\
\hline \multirow{14}{*}{ MEC } & \multirow{14}{*}{0.1} & \multirow{14}{*}{0.5} & \multirow{14}{*}{21.3} & 0 & 176.3 & 157.46 & 3 & 26.95 & 65 & 128 & 239 & 490 & 1710 \\
\hline & & & & 0.05 & 156.94 & 136.55 & 5 & 25 & 59 & 115 & 210 & 430 & 1209 \\
\hline & & & & 0.1 & 119.03 & 98.59 & 3 & 23 & 49 & 90 & 157 & 315 & 931 \\
\hline & & & & 0.2 & 66.77 & 48.04 & 3 & 19 & 33 & 53 & 85 & 161 & 470 \\
\hline & & & & 0.25 & 52.63 & 34.81 & 5 & 17 & 28 & 43 & 66 & 123 & 335 \\
\hline & & & & 0.5 & 24.75 & 10.14 & 4 & 13 & 18 & 22 & 29 & 44 & 107 \\
\hline & & & & 0.75 & 17.06 & 5.18 & 3 & 11 & 14 & 16 & 20 & 27 & 55 \\
\hline & & & & 1 & 13.39 & 3.17 & 1 & 9 & 11 & 13 & 15 & 19 & 37 \\
\hline & & & & 1.5 & 9.78 & 1.76 & 3 & 7 & 9 & 10 & 11 & 13 & 24 \\
\hline & & & & 2 & 7.9 & 1.19 & 4 & 6 & 7 & 8 & 9 & 10 & 17 \\
\hline & & & & 2.5 & 6.72 & 0.88 & 3 & 6 & 6 & 7 & 7 & 8 & 14 \\
\hline & & & & 3 & 5.92 & 0.7 & 3 & 5 & 5 & 6 & 6 & 7 & 11 \\
\hline & & & & 4 & 4.85 & 0.52 & 2 & 4 & 5 & 5 & 5 & 6 & 9 \\
\hline & & & & 5 & 4.09 & 0.35 & 2 & 4 & 4 & 4 & 4 & 5 & 7 \\
\hline
\end{tabular}


in the Phase-II data, keeping the in-control properties nearly the same as those in a normal environment, whereas the MCE chart is susceptible to changes in distribution. The EV and MEC charts are found robust under symmetric non-normal distributions. The standard deviation of RL of EV is smaller than the SDRL of MEC and MCE charts under in-control and out-of-control conditions.

\subsubsection{Limits based on non-normality}

The distributions of many quality characteristics (capacitance, insulation resistance and surface finish, roundness, mold dimensions and customer waiting times, impurity levels in semiconductor process chemicals and in nuclear reactions, and the interval between beta particle emissions) of different processes follow a non-normal distribution. Hence, the performance of the proposed EV and its competitors MEC and MCE is evaluated under different non-normal environments when the control limits are set in the same environment, unlike the previous cases. For this purpose, $t$, Laplace and Logistic from the symmetric family of distributions and Gamma and Lognormal from the skewed family of distributions are considered. Without loss of generality, parameters of the distributions are set to having mean zero and variance one for valid comparisons. The results for symmetric distributions are given in Table 12, and Table 13 contains ARL for skewed distributions.

According to Table 12, under $t$ distribution, the performance of MEC chart is similar to that of $\mathrm{EV}$ chart for shifts of small magnitude and the EV chart outperforms the MEC chart when $\delta \geq 1.5$. For small shifts in the process location, MCE is not good, but its detection ability is more efficient than that of other charts for large shifts, because when $\delta \geq 1.5$, ARLs for $\mathrm{EV}, \mathrm{MEC}$, and MCE charts are 12.29, 12.52, and 7.14, respectively. A similar type of behavior is observed for

Table 12. ARLs of mixed charts for symmetric distributions with $\mathrm{ARL}_{0} \cong 370$.

\begin{tabular}{|c|c|c|c|c|c|c|c|c|c|}
\hline \multirow{2}{*}{$\delta$} & \multicolumn{3}{|c|}{$t_{4}$} & \multicolumn{3}{|c|}{ Logistic } & \multicolumn{3}{|c|}{ Laplace } \\
\hline & EV & MEC & MCE & EV & MEC & MCE & EV & MEC & MCE \\
\hline 0 & 369.47 & 371.42 & 370.31 & 369.64 & 367.95 & 371.58 & 368.62 & 367.68 & 369.8 \\
\hline 0.25 & 72.32 & 71.12 & 140.78 & 73.04 & 71.97 & 116.48 & 73.11 & 72.36 & 130.12 \\
\hline 0.5 & 34.37 & 32.06 & 39.54 & 34.91 & 32.16 & 34.67 & 34.94 & 32.22 & 37.31 \\
\hline 0.75 & 23.4 & 21.88 & 18.02 & 23.69 & 22.01 & 16.85 & 23.7 & 22 & 17.63 \\
\hline 1 & 17.95 & 17.14 & 11.63 & 18.17 & 17.29 & 11.09 & 18.17 & 17.32 & 11.47 \\
\hline 1.5 & 12.29 & 12.52 & 7.14 & 12.47 & 12.61 & 6.83 & 12.47 & 12.71 & 7.04 \\
\hline 2 & 9.3 & 10.22 & 5.21 & 9.44 & 10.29 & 4.97 & 9.45 & 10.26 & 5.13 \\
\hline 3 & 6.32 & 7.66 & 3.44 & 6.44 & 7.71 & 3.27 & 6.44 & 7.72 & 3.38 \\
\hline 4 & 4.87 & 6.28 & 2.56 & 4.95 & 6.32 & 2.4 & 4.96 & 6.31 & 2.5 \\
\hline 5 & 4.05 & 5.35 & 2.04 & 4.11 & 5.39 & 1.96 & 4.1 & 5.4 & 2.02 \\
\hline
\end{tabular}

Table 13. ARLs of mixed charts for skewed distributions with $\mathrm{ARL}_{0} \cong 370$.

\begin{tabular}{cccccccc}
\hline \multirow{2}{*}{$\delta$} & \multicolumn{3}{c}{ Lognormal } & & \multicolumn{3}{c}{ Gamma } \\
\cline { 2 - 4 } \cline { 6 - 7 } & EV & MEC & MCE & & EV & MEC & MCE \\
\hline 0 & 374.64 & 370.71 & 372.5 & & 370.06 & 373.08 & 375.73 \\
0.25 & 74.77 & 74.17 & 177.84 & & 73.86 & 73.08 & 104.55 \\
0.5 & 33.83 & 31.21 & 65 & & 35.03 & 32.71 & 36.52 \\
0.75 & 22.79 & 20.95 & 25 & & 23.64 & 22.11 & 17.81 \\
1 & 17.35 & 16.44 & 14.6 & & 18.29 & 17.31 & 11.43 \\
1.5 & 11.9 & 12.04 & 8.55 & & 12.45 & 12.66 & 6.92 \\
2 & 8.96 & 9.74 & 6.23 & & 9.46 & 10.3 & 5.06 \\
3 & 6.1 & 7.34 & 4.15 & & 6.42 & 7.72 & 3.29 \\
4 & 4.71 & 5.92 & 3.01 & & 4.94 & 6.32 & 2.44 \\
5 & 3.89 & 4.99 & 2.58 & & 4.09 & 5.43 & 1.92 \\
\hline
\end{tabular}


other distributions such as Logistic and Laplace (cf., Table 12).

Moreover, in case of Lognormal and Gamma distributions, the EV chart performs more efficiently than the MCE chart when the value of $\delta$ is relatively small which is $\delta \leq 0.75$ and the performance of the EV is relatively better than that of the MEC chart when the value of $\delta$ is quite large as $\delta \geq 1.5$. Therefore, in general, we can say that the EV chart outperforms the MCE chart in detecting shifts of small magnitude and outperforms the MEC chart in detecting the shifts of large magnitude.

\section{An industrial application}

This section demonstrates how to construct the proposed EV chart. The dataset was supplied by Zhang and Chen [27] and Triola [28] with emphasis on the first 16 samples. In addition, each of these samples with a size of 5 produces 80 observations on the application of the proposed chart. The operation concerns the monitoring of the weights of quarters made by a mint machine, which was placed into service at U.S. Mint. The run chart, histogram, and probability plot are provided in Figures 3-5, respectively, for the behavior

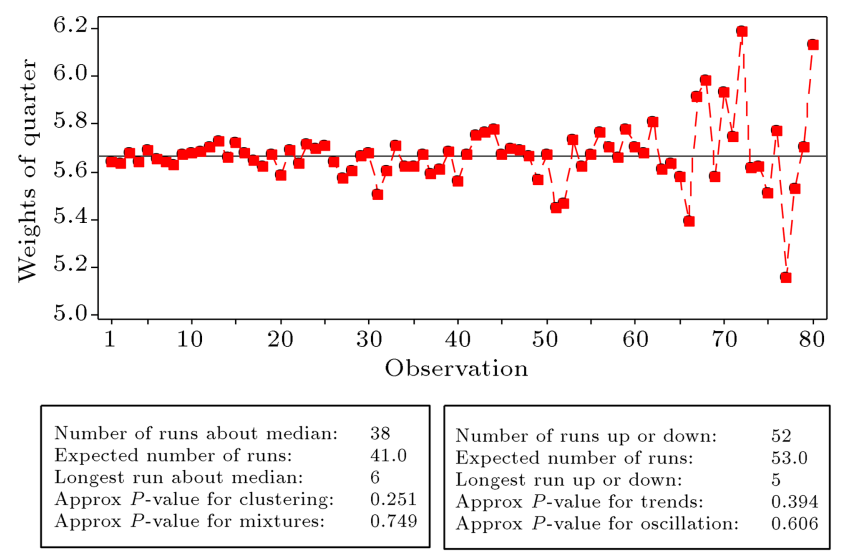

Figure 3. Run chart of weights of quarter.

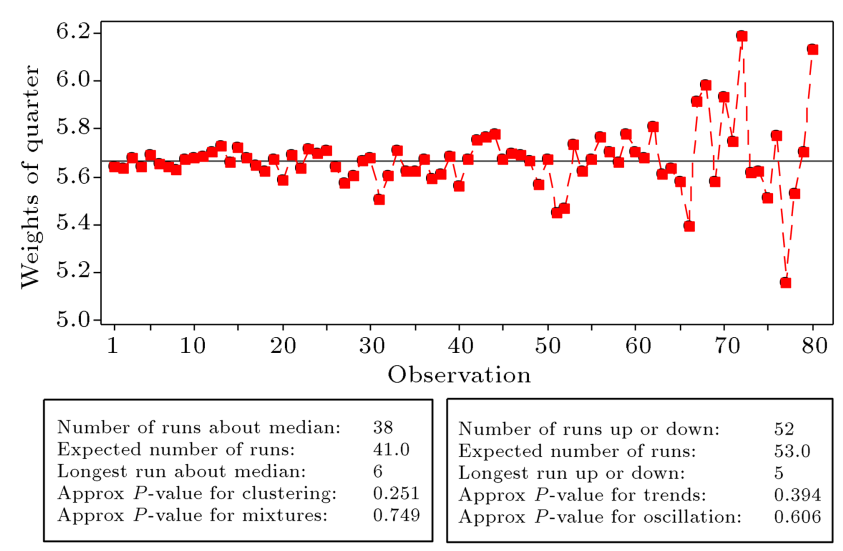

Figure 4. Histogram of weights of quarter.

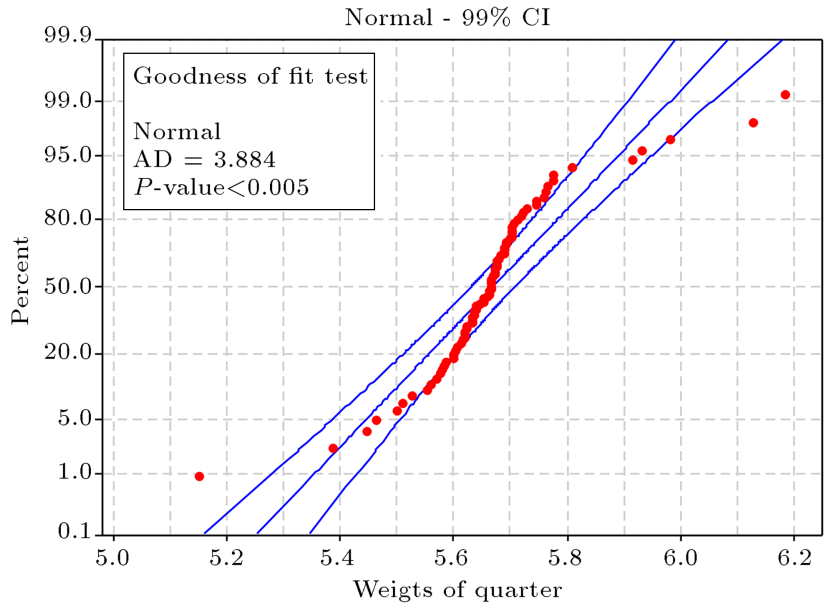

Figure 5. Probability plot of weights of quarter.

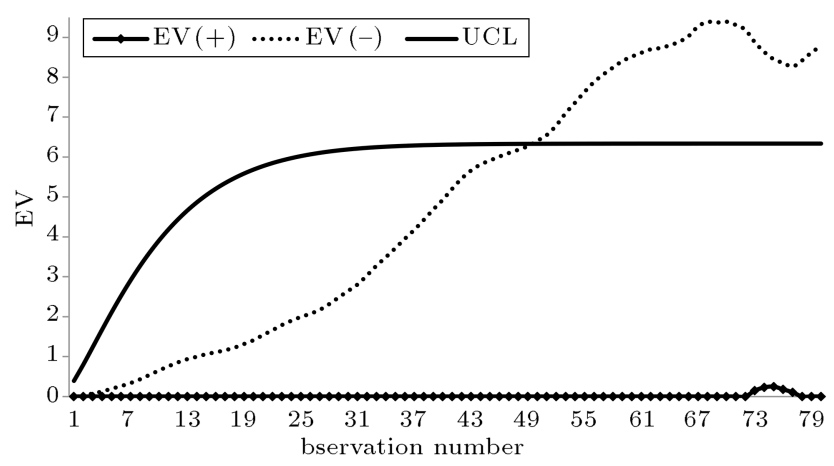

Figure 6. An industrial application of the Efficient Variant (EV) chart.

of the weights of quarters. The run chart depicts that there is more variation in the samples (50th to 80th) than that in other samples. Anderson-Darling test is applied to the identification of the distribution of the weights of quarters. The test indicates that data do not follow normal distribution as $P$-value is lower than 0.005. For comparisons, MEC and MCE charts are also constructed. The smoothing parameter is set equal to 0.10 for all the aforesaid charts and control limits are calculated to guarantee that $\mathrm{ARL}_{0} \cong 168$. The graphical displays of all three charts are presented in Figures 6-8.

The proposed chart detects a signal at the 50th sample, whereas MEC identifies the out-of-control point at the 52nd sample and MCE chart observes no out-of-control point limits. The proposed EV and MEC charts are efficient in detecting a reduction in the value of the process location parameter (cf., Figures 6 and 7), while the MCE chart shows the incapability to spot such changes in the location parameter for the same data set (cf., Figure 8).

\section{Conclusions}

Control charts are widely used in monitoring the 


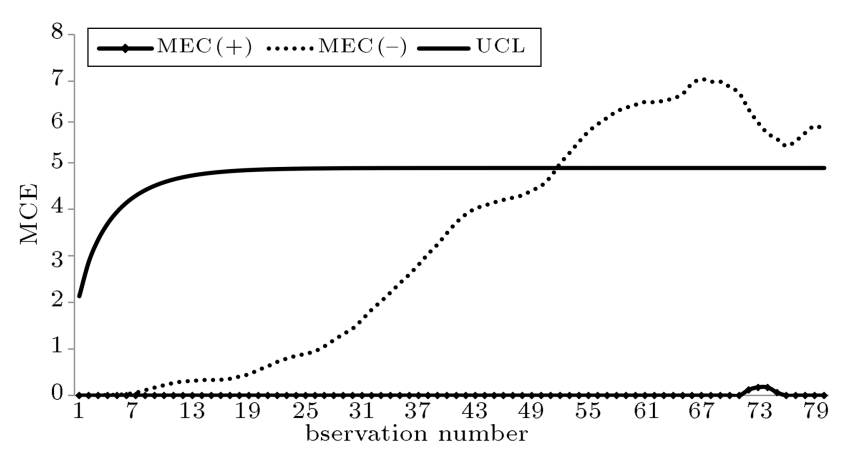

Figure 7. An industrial application of the Mixed EWMA-CUSUAM (MEC) chart.

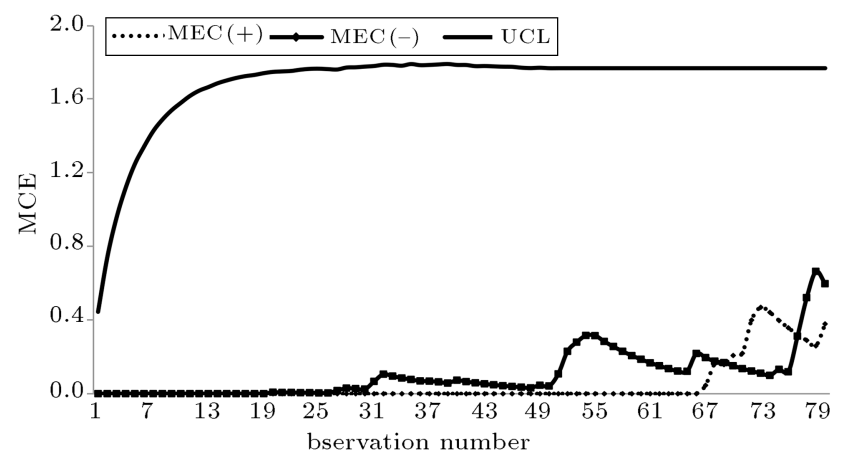

Figure 8. An industrial application of the Mixed CUSUM-EWMA (MCE) chart.

process parameters. Memory-less control charts (e.g., Shewhart-type charts) are of low efficiency in detecting the small changes in the process parameters and memory-type control charts (e.g., Cumulative Sum (CUSUM) and Exponentially Weighted Moving Average (EWMA) charts) are very sensitive to small persistent shifts. Upon enhancing the performance of CUSUM and EWMA charts, an efficient variant of memory-type charts was developed based on a mixture of the double exponentially weighted moving average chart and the CUSUM chart. The performances of the proposed efficient variant and existing counterparts (i.e., mixed EWMA-CUSUM and mixed CUSUMEWMA charts) were compared under normal and non-normal environments. The proposed variant of memory-type charts outperformed the counterparts in detecting small and moderate persistent shifts. Some features of this structure can be useful for monitoring the dispersion parameter of the process, which may be the topic of the next investigation.

\section{Acknowledgment}

The authors are thankful to the anonymous reviewers and editor for the constructive comments that helped improve the last version of the paper. The author Muhammad Riaz is indebted to King Fahd University of Petroleum and Minerals (KFUPM), Dhahran, Saudi Arabia for providing excellent research facilities.

\section{References}

1. Page, E.S. "Continuous inspection schemes", Biometrika, 41(1), pp. 100-115 (1954).

2. Roberts, S.W. "Control chart tests based on geometric moving averages", Technometrics, 3(1), pp. 239-250 (1959).

3. Abbas, N. "Homogeneously weighted moving average control chart with an application in substrate manufacturing process", Computers and Industrial Engineering, 120(6), pp. 460-470 (2018).

4. Zaman, B., Riaz, M., and Lee, M.H. "On the performance of control charts for simultaneous monitoring of location and dispersion parameters", Quality and Reliability Engineering International, 33(1), pp. 37-56 (2016).

5. Abid, M., Nazir, Z.A., Riaz, M., et al. "Use of ranked set sampling in nonparametric control charts", Journal of the Chinese Institute of Engineers, 39(5), pp. 1-19 (2016).

6. Abid, M., Nazir, Z.A., Riaz, M., et al. "Investigating the impact of ranked set sampling in nonparametric CUSUM control charts", Quality and Reliability Engineering International, 33(1), pp. 203-214 (2017).

7. Abid, M., Nazir, Z.A., Riaz, M., et al. "An efficient nonparametric EWMA Wilcoxon signed-rank chart for monitoring location", Quality and Reliability Engineering International, 33(3), pp. 665-679 (2017).

8. Lucas, J.M. "Combined Shewhart-CUSUM quality control schemes", Journal of Quality Technology, 14(2), pp. 51-59 (1982).

9. Lucas, J.M. and Saccucci, M.S. "Exponentially weighted moving average control schemes: properties and enhancements", Technometrics, 32(1), pp. 1-12 (1990).

10. Abid, M., Nazir, Z.A., Riaz, M., et al. "In-control robustness comparison of different control charts", Transaction of the Institute of Measurement and Control, 40(13), pp. 3860-3871 (2018).

11. Raza, S.M.M., Riaz, M., and Ali, S. "EWMA control chart for Poisson-exponential lifetime distribution under type I censoring", Quality and Reliability Engineering International, 32(3), pp. 995-1005 (2016).

12. Abujiya, M.R., Abbasi, S.A., and Riaz, M. "A new EWMA control chart for monitoring Poisson observations. Average control chart for monitoring the coefficient of variation", Computers and Industrial Engineering, 78, pp. 205-212 (2016).

13. Riaz, S., Riaz, M., Hussain, Z., et al. "Monitoring the performance of Bayesian EWMA control chart using loss functions", Computers and Industrial Engineering, 112, pp. 426-436 (2018).

14. Mehmood, R., Lee, M.H., Hussain, S., et al. "On efficient construction and evaluation of runs rules based control chart for known and unknown parameters under different distributions", Quality and Reliability Engineering International, 35(2), pp. 582-599 (2019). 
15. Shamma, S.E. and Shamma, A.K. "Development and evaluation of control charts using double exponentially weighted moving averages", International Journal of Quality and Reliability Management, 9(6), pp. 18-25 (1992).

16. Riaz, M., Abbas, N., and Does, R.J.M.M. "Improving the performance of CUSUM charts", Quality and Reliability Engineering International, 27(4), pp. 415424 (2011).

17. Abbas, N., Riaz, M., and Does, R.J.M.M. "Enhancing the performance of EWMA charts", Quality and Reliability Engineering International, 27(6), pp. 821-833 (2011).

18. Abbas, N., Riaz, M., and Does, R.J.M.M. "Mixed exponentially weighted moving average- cumulative sum charts for process monitoring", Quality and Reliability Engineering International, 29(3), pp. 355-365 (2013).

19. Zaman, B., Riaz, M., and Does, R.J.M.M. "Mixed cumulative sum-exponentially weighted moving average control charts: An efficient way of monitoring process location", Quality and Reliability Engineering International, 31(8), pp. 1407-1421 (2014).

20. Nazir, H.Z., Riaz, M., Does, R.J.M.M., et al. "Robust CUSUM control charting", Quality Engineering, 25(3), pp. 211-224 (2013).

21. Ahmad, L., Aslam, M., and Jun, C.H. "Designing of X-bar control charts based on process capability index using repetitive sampling", Transactions of the Institute of Measurement and Control, 36(3), pp. 367374 (2014).

22. Riaz, M., and Ali, S. "On process monitoring using location control charts under different loss functions", Transactions of the Institute of Measurement and Control, 38(9), pp. 1107-1119 (2015).

23. Abujiya, M.R., Lee, M.H., and Riaz, M. "New EWMA S2 control charts for monitoring process dispersion", Scientia Iranica, 24(1), pp. 378-389 (2017).

24. Abbasi, S.A., Ahmad, S., and Riaz, M. "On enhanced sensitivity of nonparametric EWMA control charts for process monitoring", Scientia Iranica, 24(3), pp. 16031614 (2017).

25. Ajadi, J.O. and Riaz, M. "New memory-type control charts for monitoring process mean and dispersion", Scientia Iranica, 24(6), pp. 3423-3438 (2017).

26. Hussain, S., Song, L., Ahmad, S., et al. "On auxiliary information based improved EWMA median control charts", Scientia Iranica, 25(2), pp. 954-982 (2018).

27. Zhang, L. and Chen, G. "An extended EWMA mean chart", Quality Technology and Quality Management, 2(1), pp. 39-52 (2005).

28. Triola, M.F., Elementary Statistics U sing Excel, Pearson and Addison Wesley, Boston (2004).

\section{Biographies}

Hafiz Zafar Nazir obtained his MSc and MPhil degrees in Statistics from the Department of Statistics, Quaid-i-Azam University, Islamabad, Pakistan in 2006 and 2008, respectively. He did his $\mathrm{PhD}$ in Statistics from the Institute of Business and Industrial Statistics University of Amsterdam, The Netherlands in 2014. He served as a Lecturer at the Department of Statistics, University of Sargodha, Pakistan during 20092014. He is now serving as an Assistant Professor at the Department of Statistics, University of Sargodha, Pakistan from 2015 until present. His current research interests include statistical process control, nonparametric techniques, and robust methods.

Muhammad Abid obtained his MSc and MPhil degrees in Statistics from Quaid-i-Azam University, Islamabad, Pakistan in 2008 and 2010, respectively. He did his PhD in Statistics from the Institute of Statistics, Zhejiang University, Hangzhou, China in 2017. He served as a Statistical Officer in National Accounts Wing, Pakistan Bureau of Statistics (PBS) during 2010-2011. He is now serving as an Assistant Professor at the Department of Statistics, Government College University, Faisalabad, Pakistan from 2017 to present. His research interests include statistical quality control, Bayesian statistics, non-parametric techniques, and survey sampling.

Noureen Akhtar obtained her MSc and MPhil in Statistics from Bahauddin Zakariya University, Multan, Pakistan. Currently, she is serving as an Assistant Professor at the Department of Statistics, University of Sargodha, Pakistan from 2013 until present. Her research interests include econometrics, statistical quality control, and non-parametric techniques.

Muhammad Riaz obtained his $\mathrm{PhD}$ in Statistics from the Institute for Business and Industrial Statistics, University of Amsterdam, The Netherlands in 2008. He holds the position of Professor at the Department of Mathematics and Statistics, King Fahd University of Petroleum and Minerals, Dhahran, Saudi Arabia. His current research interests include statistical process control, non-parametric techniques, and experimental design.

Sadia Qamar obtained her MSc and MPhil degrees in Statistics from the Department of Statistics, Quaid-iAzam University, Islamabad, Pakistan. She is serving as Lecturer at the Department of Statistics, University of Sargodha, Pakistan from 2009 until present. Her research interests include time series analysis, econometrics, and statistical process control. 